10,11

\title{
Удельная теплоемкость и температура Дебая сжатого кристаллического неона в модели деформируемых атомов
}

\author{
(C) Е.П. Троицкая ${ }^{1}$, Е.А. Пилипенко ${ }^{1,}$, Е.Е. Горбенко \\ ${ }^{1}$ Донецкий фризико-технический институт им. А.А. Галкина, \\ Донецк, Украина \\ 2 Луганский государственный педагогический университет, \\ Луганск, Украина \\ ฯ E-mail: pilipenko.katerina@mail.ru
}

Поступила в Редакцию 14 августа 2020 г.

В окончательной редакции 14 августа 2020 г.

Принята к публикации 14 августа 2020 г.

\begin{abstract}
Представлены теоретические $a b$ initio исследования термодинамических свойств сжатого кристалла $\mathrm{Ne}$ с учетом многочастичного взаимодействия в модели деформируемых атомов. В потенциале отталкивания наряду с трехчастичным взаимодействием, связанным с перекрытием электронных оболочек атома, учитывается деформация электронных оболочек атомов дипольного типа в парном и трехчастичном приближениях. С помощью динамической матрицы на основе неэмпирического короткодействующего потенциала отталкивания и интегрирования по десяти точкам главного значения метода Чади-Коэна проведены расчеты удельной теплоемкости и температуры Дебая в широком интервале давлений и температур. Показано, что вклады трехчастичных сил, связанных как с перекрытием электронных оболочек атомов, так и с деформацией электронных оболочек, увеличивают значение удельной теплоемкости сжатого ГЦК-Nе даже при нулевом давлении. Полученные температурные зависимости находятся в хорошем согласии с имеющимися экспериментальными данными и расчетами других авторов.
\end{abstract}

Ключевые слова: кристаллы инертных газов, трехчастичное взаимодействие, деформация электронных оболочек, фононные частоты, удельная теплоемкость, температура Дебая, высокое давление.

DOI: 10.21883/FTT.2020.12.50217.170

\section{1. Введение}

Кристаллы инертных газов (КИГ) (кроме Не) образуют ГЦК-структуру с одним атомом в элементарной ячейке. Они являются простейшими молекулярными кристаллами, которые удерживаются сравнительно слабыми силами Ван-дер-Ваальса. Благодаря этому КИГ часто используют в качестве стандартных тестовых объектов во многих теоретических исследованиях [1-3].

В отличие от тяжелых кристаллов $\mathrm{Ar}, \mathrm{Kr}$ и Хе, более легкий $\mathrm{Ne}$ проявляет свойства, отличные от других кристаллов инертных газов. Предсказано [4-7], что $\mathrm{Ne}$ имеет самую широкую энергетическую щель, самое высокое давление металлизации и противоположную зависимость плотности запрещенной щели от сжатия. Более того, $\mathrm{Ne}$ является первичной составляющей атмосфер звезд и планет [8,9]. Его поведение в экстремальных условиях важно для понимания внутренней структуры, формирования и эволюции этих астрофизических объектов. Таким образом, изучение термодинамических свойств $\mathrm{Ne}$ в условиях высоких давлений и температур представляет научный интерес.

Основная тенденция современных работ по ab initio расчетам на основе теории функционала плотности (density functional theory - DFT) заключается в стремлении как можно более точно описать слабосвязанные мо- лекулярные комплексы системы инертных газов, видоизменяя обменно-корреляционный потенциал. В качестве тестовых величин выбраны энергия связи, постоянная решетки и объемный модуль $[2,3,10]$. Предполагается, что увеличение плотности заряда в результате сжатия приведет к улучшению используемых приближений. Наиболее адекватные результаты будут получаться для тяжелых кристаллов инертных газов (в то время, как кристаллический $\mathrm{Ne}$ будет описываться наименее точно, как показано в работе [11]). По мнению авторов $[12,13]$ это связано с тем, что на основе DFT невозможно рассчитать с достаточной точностью два вида дисперсионных сил: дальнодействующее взаимодействие Ван-дерВаальса и эффекты перекрытия в короткодействующем потенциале отталкивания.

В связи с этим важно рассчитать широкий круг динамических и термодинамических характеристик сжатых КИГ из первых принципов, применяя единый подход, позволяющий описать как многочастичное взаимодействие в короткодействующем потенциале отталкивания, так и взаимодействие Ван-дер-Ваальса, которое представляет собой результат взаимного деформирующего и поляризующего действия атомов друг на друга.

В предыдущей работе [14] на основе неэмпирической версии квантово-механической модели деформируемых и поляризуемых атомов (модель Толпыго, см. [15] и 
ссылки там) построена динамическая матрица с учетом обоих видов трехчастичных взаимодействий: как за счет перекрытия электронных оболочек, так и за счет их деформации. Это позволило рассчитать для кристаллического $\mathrm{Ne}$ фононные частоты в нужных точках зоны Бриллюэна и, используя метод Чади-Коэна, энергию нулевых колебаний в широком интервале давлений.

Цель настоящей работы - рассчитать удельную теплоемкость и температуру Дебая сжатого кристаллического $\mathrm{Ne}$ и исследовать влияние трехчастичного взаимодействия в короткодействующем потенциале отталкивания и деформации электронных оболочек атомов в парном и трехчастичном приближениях на эти характеристики в широком интервале давлений и температур.

\section{2. Термодинамические свойства с учетом трехчастичного взаимодействия и деформации электронных оболочек}

Энергия связи кристалла $E_{c o h}$ и его решеточная теплоёмкость $C_{V}$ в гармоническом приближении описываются формулами:

$$
\begin{gathered}
E_{c o h}=\frac{N_{A} \Omega}{(2 \pi)^{3}} \sum_{\lambda} \int d^{3} k \hbar \omega_{\lambda}(\mathbf{k})\left[\frac{1}{2}+n_{\lambda}(\mathbf{k})\right], \\
n_{\lambda}(\mathbf{k})=\left[\exp \left(\frac{\hbar \omega_{\lambda}(\mathbf{k})}{k_{B} T}\right)-1\right]^{-1},
\end{gathered}
$$

где $N_{A}$ - число Авогадро, $\Omega=2 a^{3}$ - объем элементарной ячейки КИГ в ГЦК-фазе, $a$ - параметр решетки, равный половине ребра куба, $\omega_{\lambda}$ - частота ветви $\lambda, \mathbf{k}-$ безразмерный волновой вектор.

$$
C_{V}=\frac{R \Omega}{(2 \pi)^{3}} \sum_{\lambda} \int d^{3} k\left[\left(\frac{\hbar \omega_{\lambda}(\mathbf{k})}{k_{B} T}\right)^{2} n_{\lambda}(\mathbf{k})\left(n_{\lambda}(\mathbf{k})+1\right)\right]
$$

где $R$ - универсальная газовая постоянная, $k_{B}-$ постоянная Больцмана.

Для нахождения фононных частот в любой точке зоны Бриллюэна необходимо построить динамическую матрицу [14]. В используемой модели Толпыго помимо смещений остовов, описываемых компонентами дипольного момента $p_{\alpha}=e u_{\alpha}$, введены внутренние степени свободы $\mathbf{P}$, характеризующие состояния электронных оболочек. Поэтому для определения собственных частот $\omega_{\lambda \mathbf{k}}$, имеются две группы уравнений:

$$
\begin{aligned}
& M \omega_{\lambda \mathbf{k}}^{2} p_{\alpha}=\sum_{\beta}\left(A_{\alpha \beta} p_{\beta}+B_{\alpha \beta} P_{\beta}\right), \\
& m \omega_{\lambda \mathbf{k}}^{2} P_{\alpha}=\sum_{\beta}\left(B_{\beta \alpha}^{*} p_{\beta}+C_{\alpha \beta} P_{\beta}\right),
\end{aligned}
$$

где $M$ - масса атома, $m$ - некоторая „фиктивная““ масса порядка массы электронной оболочки, введенная исключительно для удобства расчетов, т.к. диагонализация матрицы $6 \times 6$ технически более проста, чем процедура исключения всех компонент вектора $\mathbf{P}$ из второй группы уравнений при $m=0$, как этого требует адиабатическое приближение. Таким образом, удобно ввести некоторую матрицу

$$
D=\left(\begin{array}{ll}
D^{(1)} & D^{(2)} \\
D^{(2)} & D^{(3)}
\end{array}\right)
$$

каждый элемент, которой представляет собой матрицу $3 \times 3$ :

$$
D^{(1)}=\left(\begin{array}{lll}
A_{11}(\mathbf{k}) & A_{12}(\mathbf{k}) & A_{13}(\mathbf{k}) \\
A_{21}(\mathbf{k}) & A_{22}(\mathbf{k}) & A_{23}(\mathbf{k}) \\
A_{31}(\mathbf{k}) & A_{32}(\mathbf{k}) & A_{33}(\mathbf{k})
\end{array}\right) .
$$

Аналогично для матриц $D^{(2)}\left(B_{\alpha \beta}(\mathbf{k})\right)$ и $D^{(3)}\left(C_{\alpha \beta}(\mathbf{k})\right)$. Для получения частоты нужной размерности, каждый элемент матрицы нужно умножить на размерный множитель $2 \lambda=\frac{e^{2}}{a^{3}}(e-$ заряд электрона). Тогда

$$
\begin{gathered}
A_{\alpha \beta}(\mathbf{k})=\frac{e^{2}}{a^{3}}\left[\begin{array}{c}
B \chi_{\alpha \beta}(\mathbf{k})+\delta_{\alpha \beta}\left(H \mu(\mathbf{k})+G v_{\alpha}(\mathbf{k})+F \xi(\mathbf{k})\right. \\
\left.+E \xi_{\alpha}(\mathbf{k})+V_{t} \vartheta_{\alpha}(\mathbf{k})\right)+\left(1-\delta_{\alpha \beta}\right) G \tau_{\alpha \beta}(\mathbf{k})
\end{array}\right] ; \\
B_{\alpha \beta}(\mathbf{k})=\frac{e^{2}}{a^{3}}\left[\delta_{\alpha \beta}\left(h \mu(\mathbf{k})+g v_{\alpha}(\mathbf{k})\right)+\left(1-\delta_{\alpha \beta}\right) g \tau_{\alpha \beta}(\mathbf{k})\right] ; \\
C_{\alpha \beta}(\mathbf{k})=\frac{e^{2}}{a^{3}}\left[\delta_{\alpha \beta} A^{-1}-\varphi_{\alpha \beta}(\mathbf{k})\right],
\end{gathered}
$$

где $\delta_{\alpha \beta}-$ символ Кронекера;

$$
\mu(\mathbf{k})=3-\frac{1}{2} \sum_{\gamma \neq \delta} \cos k_{\gamma} \cos k_{\delta}
$$

$$
\begin{gathered}
v_{\alpha}(\mathbf{k})=2-\cos k_{\alpha} \sum_{\gamma \neq \alpha} \cos k_{\gamma} ; \quad \tau_{\alpha \beta}(\mathbf{k})=\sin k_{\alpha} \sin k_{\beta} ; \\
\xi(\mathbf{k})=3-\sum_{\gamma} \cos 2 k_{\gamma} ; \quad \xi_{\alpha}(\mathbf{k})=1-\cos 2 k_{\alpha} ; \\
\vartheta_{\alpha}(\mathbf{k})=1-\cos k_{\alpha+1} \cos k_{\alpha+2} ; \quad \mathbf{k}=a \mathbf{K}=\pi \mathbf{q} .
\end{gathered}
$$

Здесь $H=H_{0}+\delta H, \quad G=G_{0}+\delta G, \quad$ где $H_{0}(a \sqrt{2}) \quad$ и $G_{0}(a \sqrt{2})$ являются первой и второй производными короткодействующего парного потенциала отталкивания для равновесных расстояний первых соседей; аналогично для вторых соседей $F=H_{0}(2 a)$ и $E=G_{0}(2 a) ; B$ определяет взаимодействие Ван-дер-Ваальса; $h$ и $g$ параметры деформации электронных оболочек атомов дипольного типа в парном приближении; $\chi_{\alpha \beta}(\mathbf{k})-$ функции k, происходящие от ван-дер-ваальсовских сил; $\varphi_{\alpha \beta}(\mathbf{k})$ - коэффициенты электрического поля, вызванного системой диполей $\mathbf{P}^{l} ; A-$ безразмерная поляризуемость атома. Параметры $\delta G, \delta H$ и $V_{t}$ описывают трехчастичные короткодействующие силы, обусловленные перекрытием электронных оболочек (недеформированных) атомов (см. [15] и ссылки там). 

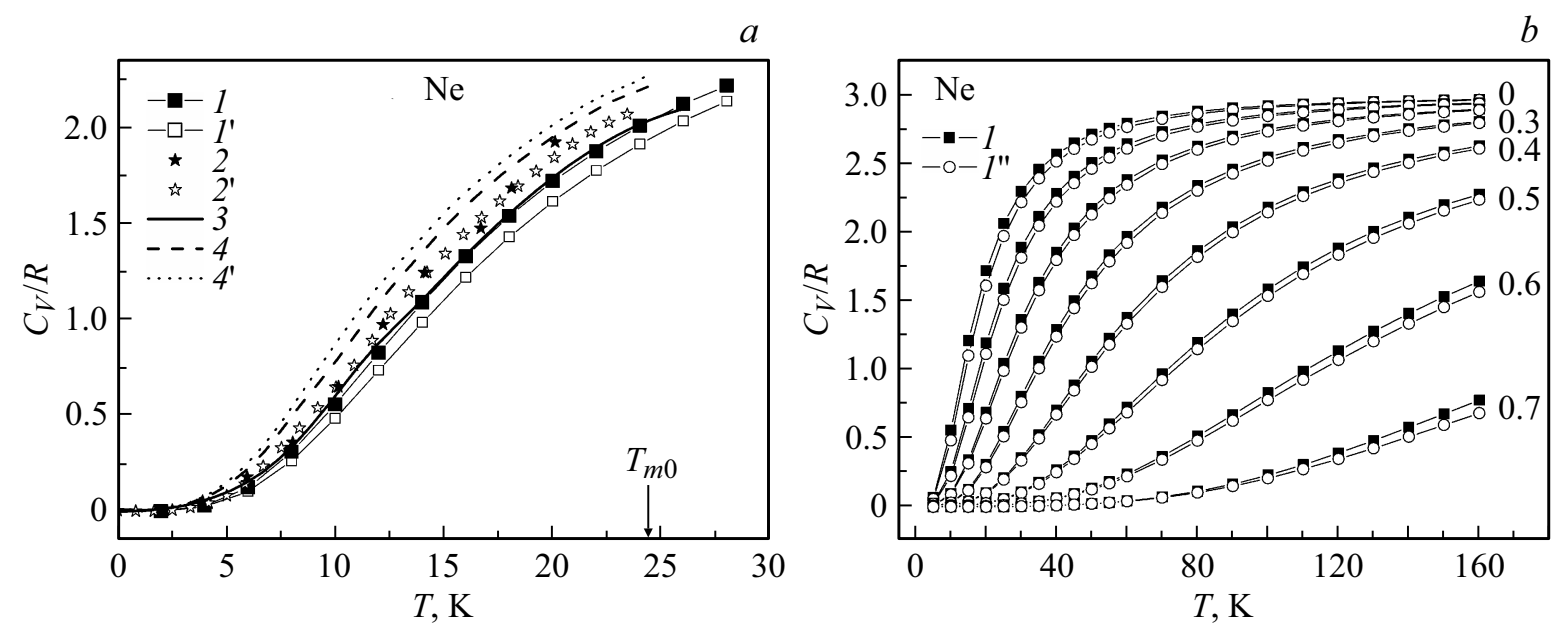

Рис. 1. Температурная зависимость теплоемкости $C_{V}(T) / R$ кристаллического Ne: $a-$ при давлении $p=u=0, b-$ при различных сжатиях от $u=0$ до 0.7. 1 - наш расчет в модели $\mathrm{MT}_{2}, 1^{\prime}$ - расчет в модели М3а [20], $1^{\prime \prime}$ - расчет в модели М3 [20]. Эксперимент: $2-[21], 2^{\prime \prime}-[22] .3-$ расчет в классической модели Толпыго (M1) [19], 4 - расчеты на основе потенциала Леннард-Джонса (LJ) [23], 4' — расчеты ELJ [23] на основе расширенного (extended) потенциала LJ (ELJ). Tемпература плавления $T_{m 0}=24.6 \mathrm{~K}$ (при $p=0$ ) показана стрелкой.

В работе [16] были рассмотрены трехчастичные силы, возникающие за счет деформации электронных оболочек атомов. В дальнейшем мы будем учитывать эти силы путем переопределения параметров $g$ и $h$. Таким образом, диагонализация динамической матрицы $D$ даст нам фононные частоты с учетом трехчастичного взаимодействия и деформации электронных оболочек в любой точке зоны Бриллюэна.

Для вычисления интегралов по зоне Бриллюэна (1) - (2) можно использовать метод Чади-Коэна [17]. Сущность этого метода состоит в замене интеграла по зоне Бриллюэна суммой значений подинтегральной функции в особых точках (точках главного значения), найденных теоретико-групповыми методами [18]. Так, в работе [19] использовались 408 точек главного значения для расчета теплоемкости и тепловых колебаний решетки КИГ при нулевом давлении в классической модели Толпыго (параметры теории определяются из минимума среднеквадратичного отклонения для $\omega_{\lambda \mathbf{k}}$ ). В работе [20] на основе двух точек главного значения метода ЧадиКоэна [17] были проведены расчеты фононных частот для кристаллического $\mathrm{Ne}$ в моделях M3 (парное взаимодействие в короткодействующем потенциале отталкивания без учета деформации электронных оболочек атома) и МЗа (парное взаимодействие и учет деформации электронных оболочек атома в парном приближении). В недавней работе [14] были рассчитаны энергия фононов и энергии нулевых колебаний в двух и десяти точках для КИГ при различных сжатиях $u=\Delta V / V_{0}$ $\left(\Delta V=V_{0}-V(p)\right.$, где $V_{0}-$ объем при $\left.p=0\right)$ на основе двух моделей: $\mathrm{MT}_{0}-\mathrm{c}$ учетом трехчастичного взаимодействия за счет перекрытия электронных оболочек и без учета деформации электронных оболочек атома и в модели $\mathrm{MT}_{2}$, которая учитывает трехчастичные силы за счет перекрытия электронных оболочек и деформацию электронных оболочек атомов дипольного типа в парном и трехчастичном приближениях.

Для количественного анализа влияния трехчастичных сил и деформации электронных оболочек на термодинамические свойства кристаллического $\mathrm{Ne}$ проведем расчеты удельной теплоемкости и температуры Дебая во всех четырех моделях $\left(\mathrm{MT}_{2}, \mathrm{MT}_{0}, \mathrm{M} 3, \mathrm{M} 3 \mathrm{a}\right)$ представленных выше.

\section{3. Удельная теплоемкость и температура Дебая}

На рис. 1, $a$ приведены экспериментальные температурные зависимости теплоемкости $C_{V}(T)$ для $\mathrm{Ne}$ при нулевом давлении $[21,22]$. Кроме того, представлены наши результаты, полученные по формуле (2) с использованием моделей $\mathrm{MT}_{2}, \mathrm{M} 3 \mathrm{a}$, расчеты в классической версии теории Толпыго (модель М1) [19], а также теоретические результаты других авторов [23].

Из рис. $1, a$ видно, что до $T \approx 10 \mathrm{~K}$ все теоретические расчеты близки между собой и хорошо согласуются с экспериментом. В области температур от $10 \mathrm{~K}$ до температуры плавления $T_{m 0}$ наилучшее согласие с экспериментом дают расчеты в модели М1 (с параметрами из [19]) и наши расчеты в модели $\mathrm{MT}_{2}$. Также близки к эксперименту расчеты [23] на основе расширенного ab initio двухчастичного потенциала Леннард-Джонса (extended Lennard-Jones potential - ELJ) [24].

Как видно из наших результатов (рис. $1, a$ ) величина $C_{V}(T) / R$, рассчитанная с учетом трехчастичных сил, возникающих за счет перекрытия электронных оболочек и деформации электронных оболочек атомов дипольного типа в парном и трехчастичном приближениях $\left(\mathrm{MT}_{2}\right)$ 

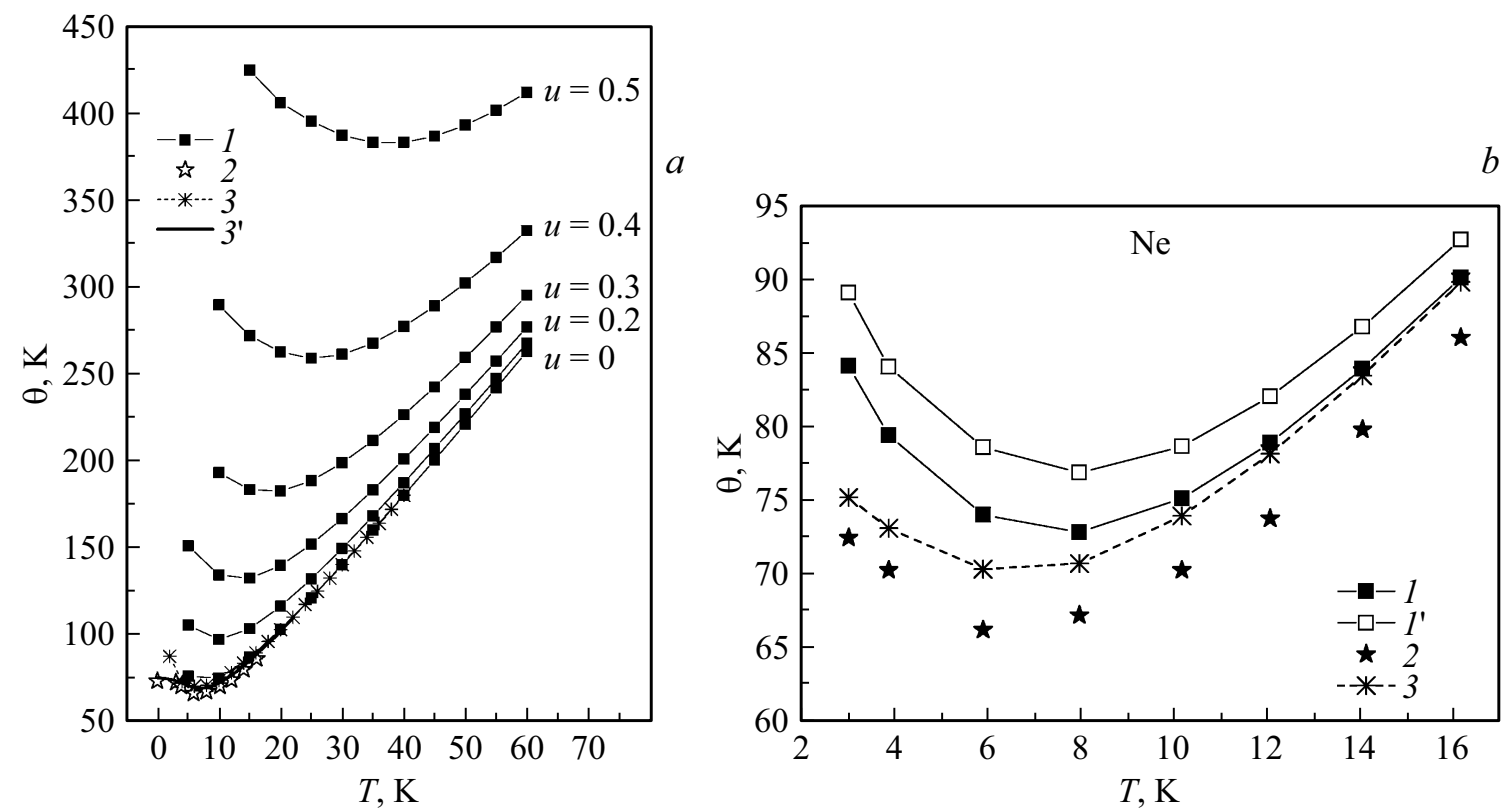

Рис. 2. Температурная зависимость $\theta(T)$ кристаллического Ne: $a-$ при разных давлениях, $b-$ при $p=0.3,3^{\prime}-$ расчеты в модели М1 по 10 [20] и 408 [19] точкам в схеме Чади-Коэна, соответственно. Остальные обозначения как на рис. 1.

практически накладывается на расчет в модели М1 (графики пересекаются в нескольких точках), приближая его к экспериментальным значениям по сравнению с расчетами в модели МЗа.

Лучшее согласие с экспериментом по теплоемкости должны давать теории, наиболее точно описывающие фононный спектр, так как $C_{V}(T)$ однозначно им определяется. Учет трехчастичных сил, обязанных своим появлением как перекрытию электронных оболочек атома, так и их деформации позволяет получить набор фононных частот [14], который дает лучшее согласие с экспериментом по теплоемкости, по сравнению с расчетами в модели МЗа, где трехчастичное взаимодействие не учитывается [25,26].

На рис. $1, b$ представлена удельная теплоемкость $\mathrm{Ne}$ при $p \neq 0$ в зависимости от температуры, рассчитанная в моделях $\mathrm{MT}_{2}$ и М3 [20]. Как видно из рис. 1, $b$, при увеличении давления значения $C_{V}$ уменьшаются в соответствующих температурных интервалах, они не достигают предельного значения 3R, а также меняется вид кривой как в одной, так и в другой модели. Видно, что с ростом сжатия трехчастичное взаимодействие и деформация электронных оболочек атомов несколько повышают значения теплоемкости даже в недеформированном кристалле $\mathrm{Ne}$ (см. рис. 1,b). Так, например, при $p=0$ и $T=160 \mathrm{~K}-C_{V}^{(\mathrm{M} 3)}=2.96 R$ и $C_{V}^{\left(\mathrm{MT}_{2}\right)}=2.97 R$; при $u=0.4(p=5.091 \mathrm{GPa})$ и $T=160 \mathrm{~K}-C_{V}^{(\mathrm{M} 3)}=2.61 R$ и $\quad C_{V}^{\left(\mathrm{MT}_{2}\right)}=2.63 R ; \quad$ при $u=0.7 \quad(p=174.86 \mathrm{GPa}) \quad$ и $T=160 \mathrm{~K}-C_{V}^{(\mathrm{M} 3)}=0.68 R$ и $C_{V}^{\left(\mathrm{MT}_{2}\right)}=0.77 R$. Такой же эффект наблюдается и с ростом температуры как без давления на кристалл, так и в сжатом состоянии. Например, для $u=0.5(p=15.64 \mathrm{GPa})$ при $T=200 \mathrm{~K}-$
$C_{V}^{(\mathrm{M} 3)}=2.48 R \quad$ и $\quad C_{V}^{\left(\mathrm{MT}_{2}\right)}=2.51 R ; \quad$ при $\quad T=400 \mathrm{~K}-$ $C_{V}^{(\mathrm{M} 3)}=2.85 R \quad$ и $C_{V}^{\left(\mathrm{MT}_{2}\right)}=2.86 R ;$ при $T=600 \mathrm{~K}-$ $C_{V}^{(\mathrm{M} 3)}=2.93 R$ и $C_{V}^{\left(\mathrm{MT}_{2}\right)}=2.94 R$.

Удельная теплоемкость $C_{V}$ мала при низких температурах $T \ll \theta_{D}\left(\theta_{D}-\right.$ температура Дебая при $\left.T=0\right)$ и пропорциональна $T^{3}$. Поэтому для сравнения теории с экспериментом удобно рассчитывать величину

$$
\theta(T)=\left(\frac{12 \pi^{4}}{5}\right)^{1 / 3} T\left(\frac{R}{C_{V}}\right)^{1 / 3}
$$

Нетрудно заметить, что при $T=0 \theta(0)=\theta_{D}$.

Температурная зависимость температуры Дебая $\theta(T)$ $\mathrm{Ne}$ при разных давлениях, которая рассчитывалась по формуле (10), представлена на рис. 2, а. Сопоставление расчетных значений температуры Дебая $\theta(T)$ при нулевом давлении с экспериментальными данными [21] показывает правильность общего хода этой зависимости в достаточно широком температурном интервале $0-20 \mathrm{~K}$ (рис. 2, $a, b)$. Это свидетельствует о том, что теория правильно предсказывает значения $\omega_{\lambda \mathbf{k}}$ на начальном участке кривых, в первую очередь, для поперечных (низких) частот.

Однако относительная погрешность наших расчетов $\theta(T)$ в моделях $\mathrm{MT}_{2}, \mathrm{MT}_{0}, \mathrm{M} 3 \mathrm{a}$ [20] и М3 по сравнению с экспериментом достаточно велика (см. таблицу) при низких температурах $T \leq 5 \mathrm{~K}$. Учет трехчастичных сил, связанных с перекрытием электронных оболочек и деформацией электронных оболочек атомов дипольного типа в парном и трехчастичном приближениях $\left(\mathrm{MT}_{2}\right)$ улучшает согласие с экспериментом и дает наименьшую погрешность (среднее значение $\gamma_{\mathrm{MT}_{2}}=9.15 \%$ ), приближая кривую температуры Дебая к экспериментальным 
Температурная зависимость $\theta(T)$ кристаллического е при $p=0$ и относительная погрешность $\gamma_{i}$

\begin{tabular}{|c|c|c|c|c|c|c|c|c|c|}
\hline \multirow{2}{*}{$T, \mathrm{~K}$} & \multicolumn{5}{|c|}{$\theta(T), \mathrm{K}$} & \multicolumn{4}{|c|}{$\gamma, \%$} \\
\hline & Эксп. [21] & $\mathrm{MT}_{2}$ & $\mathrm{MT}_{0}$ & M3a & M3 & $\gamma_{\mathrm{MT}_{2}}$ & $\gamma_{\mathrm{MT}_{0}}$ & $\gamma_{\text {МТза }}$ & $\gamma_{\mathrm{M} 3}$ \\
\hline 0 & 73.16 & - & - & - & - & - & - & - & - \\
\hline 3.01 & 72.39 & 84.052 & 84.057 & 89.028 & 89.008 & 16.11 & 16.11 & 22.98 & 22.95 \\
\hline 3.87 & 70.20 & 79.346 & 79.356 & 83.998 & 83.997 & 13.03 & 13.04 & 19.65 & 19.65 \\
\hline 5.891 & 66.15 & 73.965 & 73.989 & 78.530 & 78.554 & 11.82 & 11.86 & 18.72 & 18.76 \\
\hline 7.955 & 67.13 & 72.778 & 72.815 & 76.820 & 76.860 & 8.41 & 8.47 & 14.43 & 14.49 \\
\hline 10.148 & 70.20 & 75.072 & 75.118 & 78.593 & 78.645 & 6.94 & 7.00 & 11.95 & 12.03 \\
\hline 12.04 & 73.71 & 78.836 & 78.886 & 81.996 & 82.053 & 6.96 & 7.03 & 11.24 & 11.32 \\
\hline 14.018 & 79.74 & 83.869 & 83.920 & 86.720 & 86.780 & 5.18 & 5.25 & 8.76 & 8.83 \\
\hline 16.125 & 85.98 & 90.050 & 90.100 & 92.630 & 92.691 & 4.73 & 4.79 & 7.73 & 7.80 \\
\hline & & & & & & \multicolumn{4}{|c|}{ Среднее значение $\gamma, \%$} \\
\hline & & & & & & 9.15 & 9.19 & 14.43 & 14.48 \\
\hline
\end{tabular}

Примечание. $\gamma_{i}=\left(\left|\theta_{i}-\theta_{\exp }\right| / \theta_{\exp }\right) \cdot 100 \%$.

точкам (рис. 2,b). Модель, учитывающая трехчастичное взаимодействие за счет перекрытия электронных оболочек и не учитывающая деформацию электронных оболочек атома $\left(\mathrm{MT}_{0}\right)$ имеет близкий к предыдущему результат со средним значением $\gamma_{\mathrm{MT}_{0}}=9.19 \%$. Средние значения погрешностей моделей МЗа и М3 составляют $14.43 \%$ и $14.48 \%$ соответственно.

Большая погрешность рассчитанных нами значений $\theta(T)$ для Ne по сравнению с экспериментом при температуре $T \leq 5 \mathrm{~K}$ объясняется недостаточным числом точек Чади-Коэна [19] (см., например, кривые 3,3' на рис. $2, a)$. При температуре $T \geq 10 \mathrm{~K}$ - это недостатки, связанные с приближениями моделей $\mathrm{MT}_{2}, \mathrm{MT}_{0}, \mathrm{M} 3 \mathrm{a}$ и М3. Кроме того, необходимо принимать во внимание, что в неоне квантовые эффекты сравнительно велики,

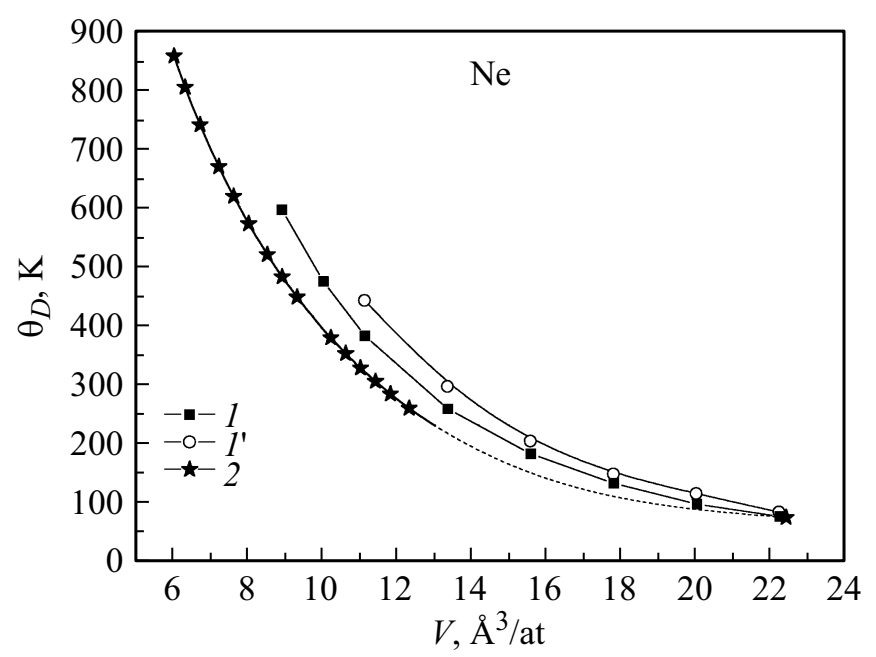

Рис. 3. Зависимость температуры Дебая от объема. 1 - наши расчеты в модели $\mathrm{MT}_{2}, 1^{\prime}$ - расчеты в модели М3 [20], 2 - эксперимент [27]. Штриховой линией обозначена проведенная нами экстраполяция к экспериментальному значению $\theta_{D}=75 \mathrm{~K}$ при $V_{0}=22.234 \AA^{3} /$ at. и модель Дебая (10), хорошо описывающая свойства в классических кристаллах, недостаточна.

На рис. 3 представлена рассчитанная нами зависимость температуры Дебая от объема до сжатия $u=0.6$ $(p=44.67 \mathrm{GPa})$ в модели $\mathrm{MT}_{2}$ и до сжатия $u=0.5$ $(p=14.3 \mathrm{GPa})$ в модели М3 [20]. Как видно, учет трехчастичного взаимодействия и деформации электронных оболочек дипольного типа в парном и трехчастичном приближениях улучшают согласие с экспериментом [27].

\section{4. Заключение}

Исследование роли трехчастичных сил и их влияния на свойства КИГ под давлением привлекает внимание многих исследователей (см., например, [12,28]). Ряд довольно важных результатов получили авторы [12], которые квантово-теоретическим методом исследовали влияние парных и трехчастичных сил, эффектов энергии нулевых колебаний и ангармонизма на уравнение состояния кристаллического Ne под давлением. В своей работе авторы [12], сравнивая результаты с экспериментальными значениями уравнения состояния, установили, что учет трехчастичного взаимодействия становится наиболее важным при высоких давлениях, а в области низкого давления доминируют энергии нулевых колебаний. Результаты [12] также подтверждают быструю сходимость разложения многочастичных сил для $\mathrm{Ne}$ даже при высоких давлениях. Влияние трехчастичных сил и ангармонических эффектов на энергию нулевых колебаний для КИГ в несжатом состоянии рассматривалось в работе [28]. Хотя авторы и не проводили сравнение своих результатов с экспериментальными, наилучший результат для $\mathrm{Ne}$ был получен в модели Эйнштейна при учете двух- и трехчастичных сил и полного учета ангармонических эффектов (погрешность при сравнении с экспериментом $\gamma=3.1 \%$ ). 
В предыдущей работе [14] в модели деформируемых и поляризуемых атомов нами была построена динамическая матрица КИГ на основе неэмпирического короткодействующего потенциала отталкивания с учетом трехчастичного взаимодействия и деформации электронных оболочек атомов дипольного типа в парном и трехчастичном приближениях. Были проведены $a b$ initio расчеты энергии фононов для сжатых кристаллов инертных газов в двух и десяти точках главного значения метода Чади-Коэна в широком интервале давлений. Для $\mathrm{Ne}$ было получено, что относительный вклад трехчастичных сил за счет перекрытия электронных оболочек по сравнению с парным имеет среднее значение $0.29 \%$ при $u=p=0$ и $0.53 \%$ при $u=0.76$ (ограничение сжатием $u=0.76$ связано с абсолютной неустойчивостью ГЦК-решетки для $\mathrm{Ne}[29])$. Учет деформации электронных оболочек дипольного типа в трехчастичном приближении наряду с трехчастичными силами, возникающими за счет перекрытия электронных оболочек, приводит к увеличению вклада обоих видов трехчастичных сил в короткодействующий потенциал отталкивания при больших сжатиях (5\% при $u=0.76$ для $\mathrm{Ne})$.

В настоящей работе на основе полученных фононных частот для кристаллического неона как под давлением, так и без него рассчитаны температурные зависимости решеточной теплоемкости и температуры Дебая, а также исследовано влияние на них трехчастичного взаимодействия в короткодействующем потенциале отталкивания и деформации электронных оболочек атомов в парном и трехчастичном приближениях. Как видно из сравнения результатов расчетов в моделях $\mathrm{MT}_{2}$ и М3а (рис. 1), вклад трехчастичных сил, обязанных своим возникновением как перекрытию, так и деформации электронных оболочек приводит к улучшению согласия с экспериментальными данными. Вклад трехчастичного взаимодействия и деформации электронных оболочек дипольного типа в парном и трехчастичном приближениях в $\mathrm{Ne}$ заметен даже при нулевом давлении, причем в том температурном интервале, где $C_{V}$ еще не приняла значение $2.99 R$, а начиная со сжатия $u=0.4(p=5.1 \mathrm{GPa})-$ во всем температурном интервале. Как показано в таблице, относительная погрешность полученных результатов по сравнению с экспериментальными данными уменьшается с увеличением температуры. Сравнение средних значений погрешностей разных расчетных моделей показывает, что вклад деформации в парном приближении уменьшает погрешность на $0.05 \%\left(\gamma_{\mathrm{M} 3 \mathrm{a}}, \gamma_{\mathrm{M} 3}\right)$, учет трехчастичного взаимодействия, связанного с перекрытием электронных оболочек, - на $0.04 \%\left(\gamma_{\mathrm{MT}_{2}}, \gamma_{\mathrm{MT}_{0}}\right)$, а вклад трехчастичных сил, как за счет перекрытия, так и связанных с деформацией $\left(\gamma_{\mathrm{MT}_{2}}, \gamma_{\mathrm{M} 3 \mathrm{a}}\right)-$ на $5.3 \%$.

Таким образом, полученные нами результаты расчета удельной теплоемкости и температуры Дебая для ГЦК-Ne с учетом трехчастичных сил и деформации электронных оболочек атомов дипольного типа в парном и трехчастичном приближении хорошо согласуются с экспериментом и очень близки к результатам расчета в классической модели Толпыго.

\section{Конфликт интересов}

Авторы заявляют, что у них нет конфликта интересов.

\section{Список литературы}

[1] I. Kwon, L.A. Collins, J.D. Kress, N. Troullier. Phys. Rev. B 52, 15165 (1995).

[2] T. Bucko, J. Hafner, S. Lebegue, J. Angyan. J.Phys. Chem. A 114, 11814 (2010).

[3] F. Tran, L. Kalantari, B. Traore, X. Rocquefelte, P. Blaha. Phys. Rev. Mater. 3, 063602 (2019).

[4] J.C. Boettger. Phys. Rev. B 33, 6788 (1986).

[5] N.D. Drummond, R.J. Needs. Phys. Rev. B 73, 024107 (2006).

[6] Y. He, X. Tang, Y. Pu. Physica B 40, 4335 (2010).

[7] J. Tang, B. Ao, L. Huang, X. Ye, Y. Gu, Q. Chen. J. Chem. Phys. 150, 111103 (2019).

[8] J. Hughto, A.S. Schneider, C.J. Horowitz, D.K. Berry. Phys. Rev. E 82, 066401 (2010).

[9] R.S. McWilliams, D.A. Dalton, Z. Konopkova, M.F. Mahmood, A.F. Goncharov. Proc. Natl. Acad. Sci. U.S.A. 112, 7925 (2015).

[10] F. Tran, P. Kovacs, L. Kalantari, G.K.H. Madsen, P. Blaha. J. Chem. Phys. 149, 144105 (2018).

[11] M.G. Medvedev, I.S. Bushmarinov, J. Sun, J.P. Perdew, K.A. Lyssenko. Science 355, 49 (2017).

[12] P. Schwerdtfeger, A. Hermann. Phys. Rev. B 80, 064106 (2009).

[13] P. Schwerdtfeger, K.G. Steenbergen, E. Pahl. Phys. Rev. B 95, 214116 (2017).

[14] Е.П. Троицкая, Е.А. Пилипенко, Е.Е. Горбенко. ФТТ 61, 1890 (2019).

[15] Е.П. Троицкая, Е.Е. Горбенко, Е.А. Пилипенко. ФНТ 42, 526 (2016).

[16] Е.П. Троицкая, В.В. Чабаненко, Е.Е. Горбенко, Е.А. Пилипенко. ФТТ 57, 114 (2015).

[17] D.J. Chadi, M.L. Cohen. Phys. Rev. B 8, 5747 (1973).

[18] A. Baldereschi. Phys. Rev. B 7, 5212 (1973).

[19] Е.В. Зароченцев, К.Б. Толпыго, Е.П. Троицкая. ФНТ 5, 11, 1324 (1979).

[20] Е.П. Троицкая, В.В. Чабаненко, Е.Е. Горбенко, Н.В. Кузовой. ФНТ 35, 8, 1041 (2009).

[21] D.N. Batchelder, D.L. Losee, R.O. Simmons. Phys. Rev. B 162, 3, 767 (1967).

[22] H. Fenichel, B. Serin. Phys. Rev. 142, 490 (1966).

[23] G.E. Moyano, P. Schwerdtfeger, K. Rosciszewski. Phys. Rev. B 75, 2, 4101 (2007).

[24] P. Schwerdtfeger, N. Gaston, R.P. Krawczyk, R. Tonner, G.E. Moyano. Phys. Rev. B 73, 6, 4112 (2006).

[25] Е.П. Троицкая, В.В. Чабаненко, Е.Е. Горбенко. ФТТ 47, 8, 1683 (2005).

[26] Е.П. Троицкая, В.В. Чабаненко, Е.Е. Горбенко. ФТТ 48, 4, 695 (2006).

[27] A. Dewaele, F. Datchi, P. Loubeyre, M. Mezouar. Phys. Rev. B 77, 094106 (2008).

[28] K. Rosciszewski, B. Paulus. Phys. Rev. B 66, 092102 (2002).

[29] Е.П. Троицкая, Е.А. Пилипенко, Е.Е. Горбенко. ФТТ 61, 154 (2019)

Редактор Е.Ю. Флегонтова 